\title{
Truncated Compound Normal with Gamma Mixture Model for Mixture Density Estimation
}

\author{
S. Viziananda Row, PhD \\ Associate Professor \\ Dept of Computer Science and \\ Systems Engg, Andhra \\ University, Visakhapatnam, \\ India
}

\author{
K. Srinivasa Rao \\ Dept. of Statistics \\ Andhra \\ University,Visakhapatnam, \\ India
}

\author{
P. Srinivasa Rao \\ Dept of Computer Science and \\ Systems Engg, Andhra \\ University,Visakhapatnam, \\ India
}

\begin{abstract}
In this paper, the truncated compound normal with gamma distribution model is formally presented and its density function has been derived for defining a mixture model(TCNGM) based on this as an extension work to the proposed compound normal with gamma mixture(CNGM) model introduced in our earlier work for image segmentation. Update equations for this model have been derived in the context of maximum likelihood estimation(MLE) procedure under Expectation Maximization(EM) framework.
\end{abstract}

\section{General Terms}

Probabilistic models, Compound Distributions, Mixture Distributions, Truncated Distributions, Maximum Likelihood Estimation, EM Framework.

\section{Keywords}

TCNGM, CNGM, NM, GM, MLE, EM

\section{INTRODUCTION}

In this paper, a formal treatment of the truncated compound normal with gamma distribution model, its density function, and a mixture model based on this(TCNGM) is presented as an extension work to the proposed compound normal with gamma mixture(CNGM) model introduced in [1],[2] for image segmentation. A truncated distribution has been introduced in [2] citing the reasons for truncation and the problems that are sometimes solved using such a distribution model.

\subsection{Compound Normal With Gamma Distribution}

As given in [3] by Normal L. Johnson et al, a compound normal with gamma distribution or Normal $\left(\mu, \sigma^{2}\right) \Lambda_{\sigma^{-2}}^{\Lambda} \operatorname{Gamma}\left(c \chi_{v}^{2}\right)$ is formed by ascribing a distribution to $\sigma^{2}$ i.e., variance by considering it as a random variable and fitting a new distribution. The corresponding distribution is defined to have a density function given as

$$
\begin{aligned}
& f(x)= \\
& (2 c)^{\frac{-v}{2}}\left[\Gamma\left(\frac{v}{2}\right)\right]^{-1} \int_{0}^{\infty}[\sqrt{2 \pi} \sigma]^{-1}\left(\sigma^{-2}\right)^{\left(\frac{v}{2}-1\right)} \cdot \exp \left[-\left(2 c \sigma^{2}\right)^{-1}\left(2 \sigma^{2}\right)^{-1}(x-\right. \\
& \left.\mu)^{2}\right] d \sigma^{-2} \\
& (1)
\end{aligned}
$$

After some mathematical transformations and further treatment, Equation (1) reduces to

$$
f(x)=\frac{1}{c^{1 / 2} B(1 / 2, v / 2)}\left[1+\frac{(x-\mu)^{2}}{c}\right]^{-(v+1) / 2}
$$

The compound normal with gamma distribution model that has been introduced has formed the basis for our work[1],[2] and a mixture model for this is used to solve the image segmentation problem.

\subsection{Truncated Distributions}

As stated in [4], truncated distributions are formed by restricting the domain of some other probability distribution. Truncated distributions are useful to solve problems where the values lie above or below a given threshold or within a specified range.

In general, if $X$ is a random variable with density $f_{x}($.$) and$ cumulative distribution $F_{x}($.$) , then the density of X$ truncated on the left at $a$ and on the right at $b$ is given by[5]

$\frac{f_{x}(x)}{F_{x}(b)-F_{x}(a)}$

For example, image segmentation problem may be viewed as mixture density estimation problem and since gray level images are spatially represented using an eight bit intensity or pixel value, the pixels only take values ranging between 0 and 255 , each representing a particular gray value ranging between black and white. This strongly suggests to define a truncated mixture model, with $0 \leq x \leq 255$ in place of the more general case of $-\infty<x<+\infty$ for the random variable $x$ that represents intensity value, for image segmentation because truncated distributions model finite range data well in comparison to the more general model.

\subsection{Mixture Distribution}

A brief introduction as given by Mood et al in [5] to the concept of contagious distribution or a mixture is given here. If $f_{0}(),. f_{1}(),. \ldots, f_{n}(),. \ldots$ is a sequence of density functions which are either all discrete density functions or all probability density functions which may or may not depend on parameters, and $p_{0}, p_{1}, \ldots, p_{n}, \ldots$ is a sequence of parameters satisfying $p_{i} \geq 0$ and $\sum_{i=0}^{\infty} p_{i}=1$, then $\sum_{i=0}^{\infty} p_{i} f_{i}(x)$ is a density function, which is sometimes called contagious distribution or a mixture.

Physical considerations of the random experiment at hand can sometimes persuade one to consider modeling the experiment with a mixture. The experimenter may know that the phenomena that he is observing are a mixture; for example, the radioactive particle emissions under observation might be a mixture of the emissions of two, or several, different types of radioactive materials [5].

For example, the current literature on statistical image segmentation techniques mostly assumes the data describing 
the image as a mixture of component distributions, as shown in Fig. 1 [6],[7],[8],[9].

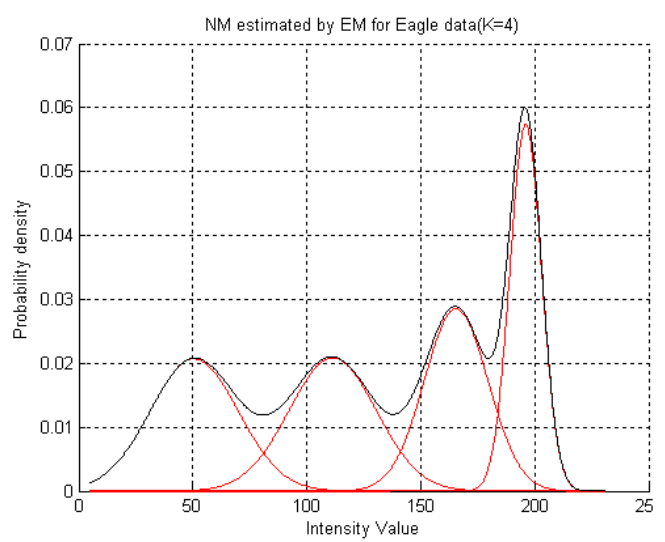

Figure 1 An Example Mixture Distribution

\subsection{Clustering As Mixture Density \\ Estimation Problem}

Several researchers have viewed clustering as mixture density estimation problem in the framework of probabilistic modeling for cluster analysis. For example, image segmentation may be thought of as a clustering problem. The current literature on statistical image segmentation techniques mostly assumes the image as of containing a mixture of components each of which following normal distribution(Normal or Gaussian Mixture) i.e., $N\left(\mu, \sigma^{2}\right)$ with some weight [6],[7],[8],[9]. In our previous work[1], we assumed the image as of containing a mixture of components each of which following compound normal with gamma distribution(CNGM)

i.e., $\operatorname{Normal}\left(\mu, \sigma^{2}\right){ }_{\sigma^{-2}}^{\Lambda} \operatorname{Gamma}\left(c \chi_{v}^{2}\right)$ with some weight. And the whole image is thought of as following the weighted distribution where weighted distribution implies weighted average of the constituent components. In that work, we have studied the feasibility of CNGM vis-a-vis normal mixture(NM) model in consideration to variations of normal distribution.

In this paper, we present the truncated version of compound normal with gamma distribution as a viable model for solving any problem that comes under the scope of cluster analysis. In particular, the scope of this paper is to describe the process of deriving the analytical expressions for model parameters in the context of maximum likelihood estimation which involved considerable mathematical rigor. The use of this model for solving image segmentation or other similar problems will be considered separately.

\section{TRUNCATED COMPOUND NORMAL WITH GAMMA MIXTURE MODEL}

We know that, for compound normal with gamma distribution, the equality that is given below holds.

$f(x)=$

$\frac{1}{c^{1 / 2} B(v / 2,1 / 2)} \int_{-\infty}^{+\infty}\left[1+\frac{(x-\mu)^{2}}{c}\right]^{-\frac{(v+1)}{2}} d x=1$

After transformations (See Appendix), the above equation may be re written as
$\frac{1}{B(1 / 2, v / 2)} \int_{0}^{1} t^{\left(\frac{v}{2}-1\right)}(1-t)^{\left(\frac{1}{2}-1\right)} d t=1$

(5)

where $t=\left[1+\frac{z^{2}}{c}\right]^{-1}=\frac{c}{c+z^{2}}$ and $z=(x-\mu)$

For the above, the cumulative distribution may be obtained [3], given a value for $z \geq 0$ or $(x-\mu) \geq 0$ (by choosing a value for $x$ ) as

$\operatorname{Pr}\left[z_{v} \leq z\right]=\operatorname{Pr}\left[z_{v} \leq 0\right]+\operatorname{Pr}\left[0 \leq z_{v} \leq z\right]$

$=\operatorname{Pr}\left[z_{v} \leq 0\right]+\frac{1}{c^{1 / 2} B(1 / 2, v / 2)} \int_{0}^{z}\left[1+\frac{y^{2}}{c}\right]^{-\frac{(v+1)}{2}} d y$

$\Rightarrow \frac{1}{2}+\frac{1}{2 B(1 / 2, v / 2)} \int_{t}^{1} w^{\left(\frac{v}{2}-1\right)}(1-w)^{\left(\frac{1}{2}-1\right)} d w$

$\Rightarrow \frac{1}{2}+\frac{1}{2}-\frac{1}{2 B(1 / 2, v / 2)} \int_{0}^{t} w^{\left(\frac{v}{2}-1\right)}(1-w)^{\left(\frac{1}{2}-1\right)} d w$

$\Rightarrow 1-\frac{1}{2} I_{t}\left(\frac{v}{2}, \frac{1}{2}\right)$

(6)

where $I_{t}\left(\frac{v}{2}, \frac{1}{2}\right)$ is incomplete beta function ratio defined as $I_{t}\left(\frac{v}{2}, \frac{1}{2}\right)=\frac{1}{B(v / 2,1 / 2)} \int_{0}^{t} w^{\left(\frac{v}{2}-1\right)}(1-w)^{\left(\frac{1}{2}-1\right)} d w$

(7)

The cumulative distribution for $z \leq 0$ or $(x-\mu) \leq 0$ is

$1-\left(1-\frac{1}{2} I_{t}\left(\frac{v}{2}, \frac{1}{2}\right)\right)=\frac{1}{2} I_{t}\left(\frac{v}{2}, \frac{1}{2}\right)$

The probability density function of Truncated Compound Normal with Gamma Mixture(TCNGM) distribution after choosing left and right truncating points as $a$ and $b$ is defined as in Equation(3) where

$f(x)=\frac{1}{c^{1 / 2} B(v / 2,1 / 2)}\left[1+\frac{(x-\mu)^{2}}{c}\right]^{-\frac{(v+1)}{2}} \quad$ is the density function defined for the compound normal with gamma distribution,

$F(b)=1-\frac{1}{2} I_{b_{1}}\left(\frac{v}{2}, \frac{1}{2}\right)$

is the cumulative distribution function for some $x$ taking value $b$ such that $x \geq \mu$, and

$F(a)=\frac{1}{2} I_{a_{1}}\left(\frac{v}{2}, \frac{1}{2}\right)$

is the cumulative distribution function for some $x$ taking value $a$ such that $x \leq \mu$.

In the Equations (9) and (10), $b_{1}=\frac{c}{c+(b-\mu)^{2}}$ and $a_{1}=$ $\frac{c}{c+(a-\mu)^{2}}$

since $t=\frac{c}{c+(x-\mu)^{2}}$

Therefore, Equation (3) may be written as

$f(x)=\frac{2}{c^{1 / 2} B(v / 2,1 / 2)\left[2-\left[I_{a_{1}}\left(\frac{v}{2}, \frac{1}{2}\right)+I_{b_{1}}\left(\frac{v}{2}, \frac{1}{2}\right)\right]\right]}\left[1+\frac{(x-\mu)^{2}}{c}\right]^{-\frac{(v+1)}{2}}$ 
or

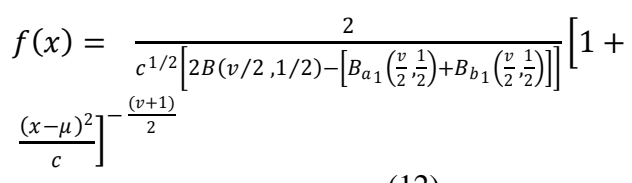

where $\quad B_{a_{1}}\left(\frac{v}{2}, \frac{1}{2}\right)$ and $B_{b_{1}}\left(\frac{v}{2}, \frac{1}{2}\right)$ are incomplete beta functions. The $f(x)$ in Equation (12) is the new density function for the truncated compound normal with gamma distribution with $a$ and $b$ as left and right truncation points.

\section{ANALYTICAL EXPRESSIONS FOR MODEL PARAMETERS, $\theta_{1}\left(\mu_{1}, c_{1}, v_{l}\right)$, FOR TCNGM}

In [1],[2], the steps involved in the maximum likelihood estimation [10] of the model parameters under Expectation Maximization framework [11] for a mixture density problem have been formally treated in the context of compound normal with gamma mixture model. In this section, the analytical expressions for the maximum likelihood estimates for model parameters, $\theta_{l}\left(\mu_{l}, c_{l}, v_{l}\right)$ which describe partly the parameter set $\Theta$ are derived in the context of the use of TCNGM under EM framework. EM algorithm optimizes the expected value of the complete data likelihood using expectation and maximization steps iteratively until convergence is reached. This optimization function is formally defined as

$Q\left(\Theta, \Theta^{g}\right)=\sum_{l=1}^{M} \sum_{i=1}^{N} \log \left(\alpha_{l} p_{l}\left(x_{i} \mid \theta_{l}\right)\right) p\left(l \mid x_{i}, \Theta^{g}\right)$

$\sum_{l=1}^{M} \sum_{i=1}^{N} \log \left(\alpha_{l}\right) p\left(l \mid x_{i}, \Theta^{g}\right)+$

$\sum_{l=1}^{M} \sum_{i=1}^{N} \log \left(p_{l}\left(x_{i} \mid \theta_{l}\right)\right) p\left(l \mid x_{i}, \Theta^{g}\right)$

where $\alpha_{l}$ is the prior probability of $l$ th component of the mixture, $p_{l}\left(x_{i} \mid \theta_{l}\right)$ is the conditional probability of $x_{i}$ belonging to $l$ and is defined for our model as in Equation (12) , and $p\left(l \mid x_{i}, \Theta^{g}\right)$ is the posterior probability of component $l$ given $x_{i}$ and current estimates of parameters $\Theta^{g}$ and is defined as

$p\left(l \mid x_{i}, \Theta^{g}\right)=\frac{\alpha_{l} p_{l}\left(x_{i} \mid \theta_{l}\right)}{\sum_{l=1}^{M} \alpha_{l} p_{l}\left(x_{i} \mid \theta_{l}\right)}$

A similar treatment is also required for the truncated version except that the modified version of the likelihood function that uses the density function defined as in Equation (12) in the previous section is used. This density function for $l$ th component is nothing but $p_{l}\left(x_{i} \mid \theta_{l}\right)$ that appears in the second term in Equation(13). Hence, in this section, the steps involved for deriving analytical expressions for $\theta_{l}\left(\mu_{l}, c_{l}, v_{l}\right)$ for the truncated version effected by the new density function are only shown.

The partial derivatives with respect to the model parameters $\mu_{l}, c_{l}$, and $v_{l}$, after equating them to zero[1],[2], are given as:

$$
\begin{aligned}
& \frac{\partial}{\partial \mu_{l}}\left[\sum_{l=1}^{M} \sum_{i=1}^{N}\left[\log \frac{2}{c_{l} 1 / 2\left[2 B\left(\frac{v_{l}}{2}, \frac{1}{2}\right)-\left[B_{a_{1}}\left(\frac{v_{l}}{2}, \frac{1}{2}\right)+B_{b_{1}}\left(\frac{v_{l}}{2}, \frac{1}{2}\right)\right]\right.}\right] 1+\right. \\
& \left.\left.\left.\frac{\left(x_{i}-\mu_{l}\right)^{2}}{c_{l}}\right]^{-\frac{\left(v_{l}+1\right)}{2}}\right] p\left(l \mid x_{i}, \Theta^{\mathrm{g}}\right)\right]=0
\end{aligned}
$$

$$
\begin{aligned}
& \frac{\partial}{\partial c_{l}}\left[\sum _ { l = 1 } ^ { M } \sum _ { i = 1 } ^ { N } \left[\log \frac{2}{c_{l}^{1 / 2}\left[2 B\left(\frac{v_{l}}{2}, \frac{1}{2}\right)-\left[B_{a_{1}}\left(\frac{v_{l}}{2}, \frac{1}{2}\right)+B_{b_{1}}\left(\frac{v_{l}}{2}, \frac{1}{2}\right)\right]\right]}[1+\right.\right. \\
& \left.\left.\left.\frac{\left(x_{i}-\mu_{l}\right)^{2}}{c_{l}}\right]^{-\frac{\left(v_{l}+1\right)}{2}}\right] p\left(l \mid x_{i}, \Theta^{\mathrm{g}}\right)\right]=0 \\
& \frac{\partial}{\partial v_{l}}\left[\sum _ { l = 1 } ^ { M } \sum _ { i = 1 } ^ { N } \left[\log \frac{2}{c_{l}^{1 / 2}\left[2 B\left(\frac{v_{l}}{2}, \frac{1}{2}\right)-\left[B_{a_{1}}\left(\frac{v_{l}}{2}, \frac{1}{2}\right)+B_{b_{1}}\left(\frac{v_{l}}{2}, \frac{1}{2}\right)\right]\right]}[1+\right.\right. \\
& \left.\left.\left.\frac{\left(x_{i}-\mu_{l}\right)^{2}}{c_{l}}\right]^{-\frac{\left(v_{l}+1\right)}{2}}\right] p\left(l \mid x_{i}, \Theta^{\mathrm{g}}\right)\right]=0
\end{aligned}
$$

Or, for component $l$, the above equations take the form as:

$$
\begin{aligned}
& \frac{\partial}{\partial \mu_{l}}\left[\sum _ { i = 1 } ^ { N } \left[\log \frac{2}{c_{l}^{1 / 2}\left[2 B\left(\frac{v_{l}}{2}, \frac{1}{2}\right)-\left[B_{a_{1}}\left(\frac{v_{l}}{2}, \frac{1}{2}\right)+B_{b_{1}}\left(\frac{v_{l}}{2}, \frac{1}{2}\right)\right]\right]}[1+\right.\right. \\
& \left.\left.\left.\frac{\left(x_{i}-\mu_{l}\right)^{2}}{c_{l}}\right]^{-\frac{\left(v_{l}+1\right)}{2}}\right] p\left(l \mid x_{i}, \Theta^{\mathrm{g}}\right)\right]=0 \\
& \frac{\partial}{\partial c_{l}}\left[\sum _ { i = 1 } ^ { N } \left[\log \frac{2}{c_{l}{ }^{1 / 2}\left[2 B\left(\frac{v_{l}}{2}, \frac{1}{2}\right)-\left[B_{a_{1}}\left(\frac{v_{l}}{2}, \frac{1}{2}\right)+B_{b_{1}}\left(\frac{v_{l}}{2}, \frac{1}{2}\right)\right]\right]}[1+\right.\right. \\
& \left.\left.\left.\frac{\left(x_{i}-\mu_{l}\right)^{2}}{c_{l}}\right]^{-\frac{\left(v_{l}+1\right)}{2}}\right] p\left(l \mid x_{i}, \Theta^{\mathrm{g}}\right)\right]=0 \\
& \frac{\partial}{\partial v_{l}}\left[\sum _ { i = 1 } ^ { N } \left[\log \frac{2}{c_{l}{ }^{1 / 2}\left[2 B\left(\frac{v_{l}}{2}, \frac{1}{2}\right)-\left[B_{a_{1}}\left(\frac{v_{l}}{2}, \frac{1}{2}\right)+B_{b_{1}}\left(\frac{v_{l}}{2}, \frac{1}{2}\right)\right]\right]}[1+\right.\right. \\
& \left.\left.\left.\frac{\left(x_{i}-\mu_{l}\right)^{2}}{c_{l}}\right]^{-\frac{\left(v_{l}+1\right)}{2}}\right] p\left(l \mid x_{i}, \Theta^{\mathrm{g}}\right)\right]=0
\end{aligned}
$$

\subsection{Derivation Of Expression For $\mu_{1}$}

Equation (18) can be rewritten as

$\sum_{i=1}^{N}\left[-\frac{\partial}{\partial \mu_{l}} \log \left[c_{l}^{1 / 2}\left[2 B\left(\frac{v_{l}}{2}, \frac{1}{2}\right)-\left[B_{a_{1}}\left(\frac{v_{l}}{2}, \frac{1}{2}\right)+\right.\right.\right.\right.$
$\left.\left.\left.\left.B_{b_{1}}\left(\frac{v_{l}}{2}, \frac{1}{2}\right)\right]\right]\right]+\frac{\partial}{\partial \mu_{l}} \log \left[1+\frac{\left(x_{i}-\mu_{l}\right)^{2}}{c_{l}}\right]^{-\frac{\left(v_{l}+1\right)}{2}}\right] p\left(l \mid x_{i}, \Theta^{\mathrm{g}}\right)=$

0

$\Rightarrow \sum_{i=1}^{N}\left[-\frac{\frac{\partial}{\partial \mu_{l}}\left[2 B\left(\frac{v_{l}}{2}, \frac{1}{2}\right)-\left[B_{a_{1}}\left(\frac{v_{l}}{2}, \frac{1}{2}\right)+B_{b_{1}}\left(\frac{v_{l}}{2}, \frac{1}{2}\right)\right]\right]}{\left[2 B\left(\frac{v_{l}}{2}, \frac{1}{2}\right)-\left[B_{a_{1}}\left(\frac{v_{l}}{2}, \frac{1}{2}\right)+B_{b_{1}}\left(\frac{v_{l}}{2}, \frac{1}{2}\right)\right]\right]}+\left(v_{l}+\right.\right.$

1) $\left.\left(\frac{x_{i}-\mu_{l}}{c_{l}}\right)\right] p\left(l \mid x_{i}, \Theta^{\mathrm{g}}\right)=0$

In Appendix, we present the details for the second 'log' term approximation and derivation of, for example, $\frac{\partial}{\partial \mu_{l}} B_{a_{1}}\left(\frac{v_{l}}{2}, \frac{1}{2}\right)$.

$\Rightarrow \sum_{i=1}^{N}\left[\frac{\frac{2}{c_{l} 1 / 2}\left[\left[\frac{c_{l}}{c_{l}+\left(a-\mu_{l}\right)^{2}}\right]^{\frac{v_{l}+1}{2}}+\left[\frac{c_{l}}{c_{l}+\left(b-\mu_{l}\right)^{2}}\right]^{\frac{v_{l}+1}{2}}\right]}{\left[2 B\left(\frac{v_{l}}{2}, \frac{1}{2}\right)-\left[B_{a_{1}}\left(\frac{v_{l}}{2}, \frac{1}{2}\right)+B_{b_{1}}\left(\frac{v_{l}}{2}, \frac{1}{2}\right)\right]\right]}+\left(v_{l}+\right.\right.$

1) $\left.\left(\frac{x_{i}-\mu_{l}}{c_{l}}\right)\right] p\left(l \mid x_{i}, \Theta^{\mathrm{g}}\right)=0$ 


$$
\begin{aligned}
& \Rightarrow \sum_{i=1}^{N}\left[\frac{2\left[\left[\frac{c_{l}}{c_{l}+\left(a-\mu_{l}\right)^{2}}\right]^{\frac{v_{l}+1}{2}}+\left[\frac{c_{l}}{c_{l}+\left(b-\mu_{l}\right)^{2}}\right]^{\frac{v_{l}+1}{2}}\right]}{c_{l}^{1 / 2}\left[2 B\left(\frac{v_{l}}{2}, \frac{1}{2}\right)-\left[B_{a_{1}}\left(\frac{v_{l}}{2}, \frac{1}{2}\right)+B_{b_{1}}\left(\frac{v_{l}}{2}, \frac{1}{2}\right)\right]\right]}+\left(v_{l}+\right.\right. \\
& \text { 1) } \left.\left(\frac{x_{i}-\mu_{l}}{c_{l}}\right)\right] p\left(l \mid x_{i}, \Theta^{\mathrm{g}}\right)=0 \\
& \Rightarrow \sum_{i=1}^{N}\left[\frac{2 c_{l}^{1 / 2}\left[\left[\frac{c_{l}}{c_{l}+\left(a-\mu_{l}\right)^{2}}\right]^{\frac{v_{l}+1}{2}}+\left[\frac{c_{l}}{c_{l}+\left(b-\mu_{l}\right)^{2}}\right]^{\frac{v_{l}+1}{2}}\right]}{\left[2 B\left(\frac{v_{l}}{2}, \frac{1}{2}\right)-\left[B_{a_{1}}\left(\frac{v_{l}}{2}, \frac{1}{2}\right)+B_{b_{1}}\left(\frac{v_{l}}{2}, \frac{1}{2}\right)\right]\right]}+\right] p\left(l \mid x_{i}, \Theta^{\mathrm{g}}\right)= \\
& 0 \Rightarrow\left(v_{l}+1\right) \mu_{l} \sum_{i=1}^{N} p\left(l \mid x_{i}, \Theta^{\mathrm{g}}\right)= \\
& \sum_{i=1}^{N}\left(\left(v_{l}+1\right) x_{i}+\right. \\
& \left.\frac{2 c_{l}^{1 / 2}\left[\left[\frac{c_{l}}{c_{l}+\left(a-\mu_{l}\right)^{2}}\right]^{\frac{v_{l}+1}{2}}+\left[\frac{c_{l}}{c_{l}+\left(b-\mu_{l}\right)^{2}}\right]^{\frac{v_{l}+1}{2}}\right]}{\left[2 B\left(\frac{v_{l}}{2}, \frac{1}{2}\right)-\left[B_{a_{1}}\left(\frac{v_{l}}{2}, \frac{1}{2}\right)+B_{b_{1}}\left(\frac{v_{l}}{2}, \frac{1}{2}\right)\right]\right]}\right) p\left(l \mid x_{i}, \Theta^{\mathrm{g}}\right)
\end{aligned}
$$$$
\Rightarrow \mu_{l} \sum_{i=1}^{N} p\left(l \mid x_{i}, \Theta^{\mathrm{g}}\right)=
$$$$
\sum_{i=1}^{N} x_{i} p\left(l \mid x_{i}, \Theta^{\mathrm{g}}\right)+
$$$$
\sum_{i=1}^{N} \frac{2 c_{l}^{1 / 2}\left[\left[\frac{c_{l}}{c_{l}+\left(a-\mu_{l}\right)^{2}}\right]^{\frac{v_{l}+1}{2}}+\left[\frac{c_{l}}{c_{l}+\left(b-\mu_{l}\right)^{2}}\right]^{\frac{v_{l}+1}{2}}\right]}{\left(2 B\left(\frac{v_{l}}{2}, \frac{1}{2}\right)-\left[B_{a_{1}}\left(\frac{v_{l}}{2}, \frac{1}{2}\right)+B_{b_{1}}\left(\frac{v_{l}}{2}, \frac{1}{2}\right)\right]\right]} p\left(l \mid x_{i}, \Theta^{\mathrm{g}}\right)
$$$$
\therefore \mu_{l}=
$$$$
\frac{\sum_{i=1}^{N} x_{i} p\left(l \mid x_{i}, \Theta^{\mathrm{g}}\right)}{\sum_{i=1}^{N} p\left(l \mid x_{i}, \Theta^{\mathrm{g}}\right)}+
$$$$
\frac{2 c_{l}^{1 / 2}\left[\left[\frac{c_{l}}{c_{l}+\left(a-\mu_{l}\right)^{2}}\right]^{\frac{v_{l}+1}{2}}+\left[\frac{c_{l}}{c_{l}+\left(b-\mu_{l}\right)^{2}}\right]^{\frac{v_{l}+1}{2}}\right]}{\left(v_{l}+1\right)\left[2 B\left(\frac{v_{l}}{2}, \frac{1}{2}\right)-\left[B_{a_{1}}\left(\frac{v_{l}}{2}, \frac{1}{2}\right)+B_{b_{1}}\left(\frac{v_{l}}{2}, \frac{1}{2}\right)\right]\right.} \frac{\sum_{i=1}^{N} p\left(l \mid x_{i}, \Theta^{\mathrm{g}}\right)}{\sum_{i=1}^{N} p\left(l \mid x_{i}, \Theta^{\mathrm{g}}\right)} \text { or }
$$$$
\mu_{l}=\frac{\sum_{i=1}^{N} x_{i} p\left(l \mid x_{i}, \Theta^{\mathrm{g}}\right)}{\sum_{i=1}^{N} p\left(l \mid x_{i}, \Theta^{g}\right)}+\frac{2 c_{l}^{1 / 2}\left[\left[\frac{c_{l}}{c_{l}+\left(a-\mu_{l}\right)^{2}}\right]^{\frac{v_{l}+1}{2}}+\left[\frac{c_{l}}{c_{l}+\left(b-\mu_{l}\right)^{2}}\right]^{\frac{v_{l}+1}{2}}\right]}{\left(v_{l}+1\right) B\left(\frac{v_{l}}{2}, \frac{1}{2}\right)\left[2-\left[I_{a_{1}}\left(\frac{v_{l}}{2}, \frac{1}{2}\right)+I_{b_{1}}\left(\frac{v_{l}}{2}, \frac{1}{2}\right)\right]\right]}
$$

\subsection{Derivation Of Expression For $\mathbf{c}_{\mathbf{l}}$}

Equation (19) can be rewritten as

$\sum_{i=1}^{N}\left[-\frac{\partial}{\partial c_{l}} \frac{\log c_{l}}{2}-\frac{\partial}{\partial c_{l}} \log \left[2 B\left(\frac{v_{l}}{2}, \frac{1}{2}\right)-\left[B_{a_{1}}\left(\frac{v_{l}}{2}, \frac{1}{2}\right)+\right.\right.\right.$

$\left.\left.\left.B_{b_{1}}\left(\frac{v_{l}}{2}, \frac{1}{2}\right)\right]\right]+\frac{\partial}{\partial c_{l}} \log \left[1+\frac{\left(x_{i}-\mu_{l}\right)^{2}}{c_{l}}\right]^{-\frac{\left(v_{l}+1\right)}{2}}\right] p\left(l \mid x_{i}, \Theta^{\mathrm{g}}\right)=0$

$\sum_{i=1}^{N}\left[-\frac{1}{2 c_{l}}-\frac{\frac{\partial}{\partial c_{l}}\left[2 B\left(\frac{v_{l}}{2}, \frac{1}{2}\right)-\left[B_{a_{1}}\left(\frac{v_{l}}{2}, \frac{1}{2}\right)+B_{b_{1}}\left(\frac{v_{l}}{2}, \frac{1}{2}\right)\right]\right]}{\left[2 B\left(\frac{v_{l}}{2}, \frac{1}{2}\right)-\left[B_{a_{1}}\left(\frac{v_{l}}{2}, \frac{1}{2}\right)+B_{b_{1}}\left(\frac{v_{l}}{2}, \frac{1}{2}\right)\right]\right]}+\frac{\partial}{\partial c_{l}} \log [1+\right.$ $\left.\left.\frac{\left(x_{i}-\mu_{l}\right)^{2}}{c_{l}}\right]^{-\frac{\left(v_{l}+1\right)}{2}}\right] p\left(l \mid x_{i}, \Theta^{\mathrm{g}}\right)=0$

The details of the second ' $\log$ ' term approximation and derivation of, for example, $\frac{\partial}{\partial c_{l}} B_{a_{1}}\left(\frac{v_{l}}{2}, \frac{1}{2}\right)$ are presented in Appendix. Therefore, the above equation changes to

$$
\begin{aligned}
& \sum_{i=1}^{N}\left[-\frac{1}{2 c_{l}}+\frac{\left[\left(a-\mu_{l}\right)\left[\frac{c_{l}}{c_{l}+\left(a-\mu_{l}\right)^{2}}\right]^{\frac{v_{l}+1}{2}}+\left(b-\mu_{l}\right)\left[\frac{c_{l}}{c_{l}+\left(b-\mu_{l}\right)^{2}}\right]^{\frac{v_{l}+1}{2}}\right]}{c_{l} c_{l}^{1 / 2}\left[2 B\left(\frac{v_{l}}{2}, \frac{1}{2}\right)-\left[B_{a_{1}}\left(\frac{v_{l}}{2}, \frac{1}{2}\right)+B_{b_{1}}\left(\frac{v_{l}}{2}, \frac{1}{2}\right)\right]\right]}+\right. \\
& \left.\frac{\left(v_{l}+1\right)\left(x_{i}-\mu_{l}\right)^{2}}{2 c_{l}^{2}}\right] p\left(l \mid x_{i}, \Theta^{\mathrm{g}}\right)=0
\end{aligned}
$$$$
\Rightarrow \sum_{i=1}^{N}\left[1-\frac{2\left[\left(a-\mu_{l}\right)\left[\frac{c_{l}}{c_{l}+\left(a-\mu_{l}\right)^{2}}\right]^{\frac{v_{l}+1}{2}}+\left(b-\mu_{l}\right)\left[\frac{c_{l}}{c_{l}+\left(b-\mu_{l}\right)^{2}}\right]^{\frac{v_{l}+1}{2}}\right]}{c_{l}^{1 / 2}\left[2 B\left(\frac{v_{l}}{2}, \frac{1}{2}\right)-\left[B_{a_{1}}\left(\frac{v_{l}}{2}, \frac{1}{2}\right)+B_{b_{1}}\left(\frac{v_{l}}{2}, \frac{1}{2}\right)\right]\right]}-\right.
$$$$
\left.\frac{\left(v_{l}+1\right)\left(x_{i}-\mu_{l}\right)^{2}}{c_{l}}\right] p\left(l \mid x_{i}, \Theta^{\mathrm{g}}\right)=0 \text {, or }
$$$$
\sum_{i=1}^{N}\left[c_{l}-\frac{2 c_{l}^{1 / 2}\left[\left(a-\mu_{l}\right)\left[\frac{c_{l}}{c_{l}+\left(a-\mu_{l}\right)^{2}}\right]^{\frac{v_{l}+1}{2}}+\left(b-\mu_{l}\right)\left[\frac{c_{l}}{c_{l}+\left(b-\mu_{l}\right)^{2}}\right]^{\frac{v_{l}+1}{2}}\right]}{\left[2 B\left(\frac{v_{l}}{2}, \frac{1}{2}\right)-\left[B_{a_{1}}\left(\frac{v_{l}}{2}, \frac{1}{2}\right)+B_{b_{1}}\left(\frac{v_{l}}{2}, \frac{1}{2}\right)\right]\right]}-\right.
$$$$
\left.\left(v_{l}+1\right)\left(x_{i}-\mu_{l}\right)^{2}\right] p\left(l \mid x_{i}, \Theta^{\mathrm{g}}\right)=0
$$

$c_{l}-$

$\left.\frac{2 c_{l}^{1 / 2}\left[\left(a-\mu_{l}\right)\left[\frac{c_{l}}{c_{l}+\left(a-\mu_{l}\right)^{2}}\right]^{\frac{v_{l}+1}{2}}+\left(b-\mu_{l}\right)\left[\frac{c_{l}}{c_{l}+\left(b-\mu_{l}\right)^{2}}\right]^{\frac{v_{l}+1}{2}}\right]}{\left[2 B\left(\frac{v_{l}}{2}, \frac{1}{2}\right)-\left[B_{a_{1}}\left(\frac{v_{l}}{2}, \frac{1}{2}\right)+B_{b_{1}}\left(\frac{v_{l}}{2}, \frac{1}{2}\right)\right]\right]}\right] \sum_{i=1}^{N} p\left(l \mid x_{i}, \Theta^{\mathrm{g}}\right)$

$$
\begin{aligned}
& =\left(v_{l}+1\right) \sum_{i=1}^{N}\left(x_{i}-\mu_{l}\right)^{2} p\left(l \mid x_{i}, \Theta^{\mathrm{g}}\right) \\
& \Rightarrow c_{l}-\frac{2 c_{l}^{1 / 2}\left[\left(a-\mu_{l}\right)\left[\frac{c_{l}}{c_{l}+\left(a-\mu_{l}\right)^{2}}\right]^{\frac{v_{l}+1}{2}}+\left(b-\mu_{l}\right)\left[\frac{c_{l}}{c_{l}+\left(b-\mu_{l}\right)^{2}}\right]^{\frac{v_{l}+1}{2}}\right]}{\left[2 B\left(\frac{v_{l}}{2}, \frac{1}{2}\right)-\left[B_{a_{1}}\left(\frac{v_{l}}{2}, \frac{1}{2}\right)+B_{b_{1}}\left(\frac{v_{l}}{2}, \frac{1}{2}\right)\right]\right]}=
\end{aligned}
$$$$
\frac{\left(v_{l}+1\right) \sum_{i=1}^{N}\left(x_{i}-\mu_{l}\right)^{2} p\left(l \mid x_{i}, \Theta^{\mathrm{g}}\right)}{\sum^{N}\left(l \mid x_{i}, \Theta^{\mathrm{g}}\right)}
$$

$$
\therefore c_{l}=
$$

$\frac{\left(v_{l}+1\right) \sum_{i=1}^{N}\left(x_{i}-\mu_{l}\right)^{2} p\left(l \mid x_{i}, \Theta^{\mathrm{g}}\right)}{\sum_{i=1}^{N} p\left(l \mid x_{i}, \Theta^{\mathrm{g}}\right)}+$

$$
\frac{2 c_{l}^{1 / 2}\left[\left(a-\mu_{l}\right)\left[\frac{c_{l}}{c_{l}+\left(a-\mu_{l}\right)^{2}}\right]^{\frac{v_{l}+1}{2}}+\left(b-\mu_{l}\right)\left[\frac{c_{l}}{c_{l}+\left(b-\mu_{l}\right)^{2}}\right]^{\frac{v_{l}+1}{2}}\right]}{\left[2 B\left(\frac{v_{l}}{2}, \frac{1}{2}\right)-\left[B_{a_{1}}\left(\frac{v_{l}}{2}, \frac{1}{2}\right)+B_{b_{1}}\left(\frac{v_{l}}{2}, \frac{1}{2}\right)\right]\right]} \text { or }
$$

$c_{l}=$

$$
\begin{aligned}
& \frac{\left(v_{l}+1\right) \sum_{i=1}^{N}\left(x_{i}-\mu_{l}\right)^{2} p\left(l \mid x_{i}, \Theta^{\mathrm{g}}\right)}{\sum_{i=1}^{N} p\left(l \mid x_{i}, \Theta^{\mathrm{g}}\right)}+ \\
& \frac{2 c_{l}^{1 / 2}\left[\left(a-\mu_{l}\right)\left[\frac{c_{l}}{c_{l}+\left(a-\mu_{l}\right)^{2}}\right]^{\frac{v_{l}+1}{2}}+\left(b-\mu_{l}\right)\left[\frac{c_{l}}{c_{l}+\left(b-\mu_{l}\right)^{2}}\right]^{\frac{v_{l}+1}{2}}\right]}{B\left(\frac{v_{l}}{2}, \frac{1}{2}\right)\left[2-\left[I_{a_{1}}\left(\frac{v_{l}}{2}, \frac{1}{2}\right)+I_{b_{1}}\left(\frac{v_{l}}{2}, \frac{1}{2}\right)\right]\right]}
\end{aligned}
$$

\subsection{Derivation Of Expression For $v_{\mathbf{l}}$}

Equation (20) can be rewritten as 


$$
\begin{aligned}
& \sum_{i=1}^{N}\left[-\frac{\partial}{\partial v_{l}} \log \left[2 B\left(\frac{v_{l}}{2}, \frac{1}{2}\right)-\left[B_{a_{1}}\left(\frac{v_{l}}{2}, \frac{1}{2}\right)+B_{b_{1}}\left(\frac{v_{l}}{2}, \frac{1}{2}\right)\right]\right]-\right. \\
& \left.\frac{\partial}{\partial v_{l}}\left(\frac{v_{l}}{2}\right) \log \left[1+\frac{\left(x_{i}-\mu_{l}\right)^{2}}{c_{l}}\right]\right] p\left(l \mid x_{i}, \Theta^{\mathrm{g}}\right)=0 \\
& \Rightarrow \sum_{i=1}^{N}\left[\frac{\frac{\partial}{\partial v_{l}}\left[2 B\left(\frac{v_{l}}{2}, \frac{1}{2}\right)-\left[B_{a_{1}}\left(\frac{v_{l}}{2}, \frac{1}{2}\right)+B_{b_{1}}\left(\frac{v_{l}}{2}, \frac{1}{2}\right)\right]\right]}{\left[2 B\left(\frac{v_{l}}{2}, \frac{1}{2}\right)-\left[B_{a_{1}}\left(\frac{v_{l}}{2}, \frac{1}{2}\right)+B_{b_{1}}\left(\frac{v_{l}}{2}, \frac{1}{2}\right)\right]\right]}+\frac{1}{2} \log [1+\right. \\
& \left.\left.\frac{\left(x_{i}-\mu_{l}\right)^{2}}{c_{l}}\right]\right] p\left(l \mid x_{i}, \Theta^{\mathrm{g}}\right)=0
\end{aligned}
$$

Please see Appendix for the proof for $\frac{\partial}{\partial v_{l}} B\left(\frac{v_{l}}{2}, \frac{1}{2}\right)$.

Therefore, the above equation changes to

$$
\begin{aligned}
& \sum_{i=1}^{N}\left[\frac{\left[2\left(-\frac{1}{2} B\left(\frac{v_{l}}{2}, \frac{3}{2}\right)\right)-\left[-\frac{1}{2} B_{a_{1}}\left(\frac{v_{l}}{2}, \frac{3}{2}\right)+\left(-\frac{1}{2} B_{b_{1}}\left(\frac{v_{l}}{2}, \frac{3}{2}\right)\right)\right]\right.}{\left[2 B\left(\frac{v_{l}}{2}, \frac{1}{2}\right)-\left[B_{a_{1}}\left(\frac{v_{l}}{2}, \frac{1}{2}\right)+B_{b_{1}}\left(\frac{v_{l}}{2}, \frac{1}{2}\right)\right]\right]}+\frac{1}{2} \log [1+\right. \\
& \left.\left.\frac{\left(x_{i}-\mu_{l}\right)^{2}}{c_{l}}\right]\right] p\left(l \mid x_{i}, \Theta^{\mathrm{g}}\right)=0
\end{aligned}
$$

$\Rightarrow$

$$
\begin{aligned}
& \sum_{i=1}^{N}\left[\frac{-\left[2 B\left(\frac{v_{l}}{2}, \frac{3}{2}\right)-B\left(\frac{v_{l}}{2}, \frac{3}{2}\right)\left[I_{a_{1}}\left(\frac{v_{l}}{2}, \frac{3}{2}\right)+I_{b_{1}}\left(\frac{v_{l}}{2}, \frac{3}{2}\right)\right]\right]}{2\left[2 B\left(\frac{v_{l}}{2}, \frac{1}{2}\right)-B\left(\frac{v_{l}}{2}, \frac{1}{2}\right)\left[I_{a_{1}}\left(\frac{v_{l}}{2}, \frac{1}{2}\right)+I_{b_{1}}\left(\frac{v_{l}}{2}, \frac{1}{2}\right)\right]\right]}+\frac{1}{2} \log [1+\right. \\
& \left.\left.\frac{\left(x_{i}-\mu_{l}\right)^{2}}{c_{l}}\right]\right] p\left(l \mid x_{i}, \Theta^{\mathrm{g}}\right)=0 \\
& \because \frac{\partial}{\partial v_{l}} B_{a_{1}}\left(\frac{v_{l}}{2}, \frac{1}{2}\right)=-\frac{1}{2} B_{a_{1}}\left(\frac{v_{l}}{2}, \frac{3}{2}\right)
\end{aligned}
$$

for which, the proof is given in Appendix

$$
\begin{aligned}
& \Rightarrow \sum_{i=1}^{N}\left[\frac{-\mathrm{B}\left(\frac{v_{l}}{2}, \frac{3}{2}\right)\left[2-\left[I_{a_{1}}\left(\frac{v_{l}}{2}, \frac{3}{2}\right)+I_{b_{1}}\left(\frac{v_{l}}{2}, \frac{3}{2}\right)\right]\right]}{\mathrm{B}\left(\frac{v_{l}}{2}, \frac{1}{2}\right)\left[2-\left[I_{a_{1}}\left(\frac{v_{l}}{2}, \frac{1}{2}\right)+I_{b_{1}}\left(\frac{v_{l}}{2}, \frac{1}{2}\right)\right]\right]}+\log [1+\right. \\
& \left.\left.\frac{\left(x_{i}-\mu_{l}\right)^{2}}{c_{l}}\right]\right] p\left(l \mid x_{i}, \Theta^{\mathrm{g}}\right)=0
\end{aligned}
$$

The above equation further leads to

$$
\begin{aligned}
& \sum_{i=1}^{N}\left[\frac{-\left[2-\left[I_{a_{1}}\left(\frac{v_{l}}{2}, \frac{3}{2}\right)+I_{b_{1}}\left(\frac{v_{l}}{2}, \frac{3}{2}\right)\right]\right]}{\left(v_{l}+1\right)\left[2-\left[I_{a_{1}}\left(\frac{v_{l}}{2}, \frac{1}{2}\right)+I_{b_{1}}\left(\frac{v_{l}}{2}, \frac{1}{2}\right)\right]\right]}+\right. \\
& \left.\log \left[1+\frac{\left.\left(x_{i}-\mu_{l}\right)^{2}\right]}{c_{l}}\right]\right] p\left(l \mid x_{i}, \Theta^{\mathrm{g}}\right)=0 \\
& \because \frac{\mathrm{B}\left(\frac{v_{l}}{2}, \frac{3}{2}\right)}{\mathrm{B}\left(\frac{v_{l}}{2}, \frac{1}{2}\right)}=\frac{1}{\left(v_{l}+1\right)} \text { Please refer to Appendix for the proof. }
\end{aligned}
$$

The above equation may be written as

$$
\begin{aligned}
& \left(v_{l}+1\right) \sum_{i=1}^{N} \log \left[1+\frac{\left(x_{i}-\mu_{l}\right)^{2}}{c_{l}}\right] p\left(l \mid x_{i}, \Theta^{\mathrm{g}}\right)= \\
& \frac{\left[2-\left[I_{a_{1}}\left(\frac{v_{l}}{2}, \frac{3}{2}\right)+I_{b_{1}}\left(\frac{v_{l}}{2}, \frac{3}{2}\right)\right]\right]}{\left[2-\left[I_{a_{1}}\left(\frac{v_{l}}{2}, \frac{1}{2}\right)+I_{b_{1}}\left(\frac{v_{l}}{2}, \frac{1}{2}\right)\right]\right]} \sum_{i=1}^{N} p\left(l \mid x_{i}, \Theta^{\mathrm{g}}\right) \\
& \therefore v_{l}=\frac{\left[2-\left[I_{a_{1}}\left(\frac{v_{l}}{2}, \frac{3}{2}\right)+I_{b_{1}}\left(\frac{v_{l}}{2}, \frac{3}{2}\right)\right]\right]}{\left[2-\left[I_{a_{1}}\left(\frac{v_{l}}{2}, \frac{1}{2}\right)+I_{b_{1}}\left(\frac{v_{l}}{2}, \frac{1}{2}\right)\right]\right]} \frac{\sum_{i=1}^{N} p\left(l \mid x_{i}, \Theta^{\mathrm{g}}\right)}{\sum_{i=1}^{N} \log \left[1+\frac{\left(x_{i}-\mu_{l}\right)^{2}}{c_{l}}\right] p\left(l \mid x_{i}, \Theta^{\mathrm{g}}\right)}-1
\end{aligned}
$$

Therefore, the update equations for $\mu_{l}, c_{l}$, and $v_{l}$ after solving Equations (18), (19), and (20) are
$\mu_{l}=\frac{\sum_{i=1}^{N} x_{i} p\left(l \mid x_{i}, \Theta^{g}\right)}{\sum_{i=1}^{N} p\left(l \mid x_{i}, \Theta^{g}\right)}+\frac{2 c_{l}^{1 / 2}\left[\left[\frac{c_{l}}{c_{l}+\left(a-\mu_{l}\right)^{2}}\right]^{\frac{v_{l}+1}{2}}+\left[\frac{c_{l}}{c_{l}+\left(b-\mu_{l}\right)^{2}}\right]^{\frac{v_{l}+1}{2}}\right]}{\left(v_{l}+1\right) B\left(\frac{v_{l}}{2}, \frac{1}{2}\right)\left[2-\left[I_{a_{1}}\left(\frac{v_{l}}{2}, \frac{1}{2}\right)+I_{b_{1}}\left(\frac{v_{l}}{2}, \frac{1}{2}\right)\right]\right]}$

$c_{l}=$

$\frac{\left(v_{l}+1\right) \sum_{i=1}^{N}\left(x_{i}-\mu_{l}\right)^{2} p\left(l \mid x_{i}, \Theta^{\mathrm{g}}\right)}{\sum^{N} p\left(l \mid x_{i} \Theta^{\mathrm{g}}\right)}+$

$\frac{2 c_{l}^{1 / 2}\left[\left(a-\mu_{l}\right)\left[\frac{c_{l}}{c_{l}+\left(a-\mu_{l}\right)^{2}}\right]^{\frac{v_{l}+1}{2}}+\left(b-\mu_{l}\right)\left[\frac{c_{l}}{c_{l}+\left(b-\mu_{l}\right)^{2}}\right]^{\frac{v_{l}+1}{2}}\right]}{B\left(\frac{v_{l}}{2}, \frac{1}{2}\right)\left[2-\left[I_{a_{1}}\left(\frac{v_{l}}{2}, \frac{1}{2}\right)+I_{b_{1}}\left(\frac{v_{l}}{2}, \frac{1}{2}\right)\right]\right]}$

$v_{l}=\frac{\left[2-\left[I_{a_{1}}\left(\frac{v_{l}}{2}, \frac{3}{2}\right)+I_{b_{1}}\left(\frac{v_{l}}{2}, \frac{3}{2}\right)\right]\right]}{\left[2-\left[I_{a_{1}}\left(\frac{v_{l}}{2}, \frac{1}{2}\right)+I_{b_{1}}\left(\frac{v_{l}}{2}, \frac{1}{2}\right)\right]\right]} \frac{\sum_{i=1}^{N} p\left(l \mid x_{i}, \Theta^{\mathrm{g}}\right)}{\sum_{i=1}^{N} \log \left[1+\frac{\left(x_{i}-\mu_{l}\right)^{2}}{c_{l}}\right] p\left(l \mid x_{i}, \Theta^{\mathrm{g}}\right)}-1$

\section{CONCLUSIONS}

In this paper, a formal treatment of mixture density estimation procedure for the truncated compound normal with gamma mixture model is presented. The analytical expressions for the maximum likelihood estimates for model parameters, $\theta_{l}\left(\mu_{l}, c_{l}, v_{l}\right)$ which describe partly the parameter set $\Theta$, have been derived since the derivation for these parameters involved some added complexity than that for the un truncated one. The derived expressions are similar in form to compound normal with gamma mixture model except that these include some additional terms due to the truncation done with respect to the left and right truncation defined by 'a' and ' $b$ ' respectively. These expressions can be embedded into the Expectation Maximization framework for solving mixture density estimation problem. The EM framework for this truncated mixture model that can be used to solve mixture density estimation problems like image segmentation and other clustering problems is considered as an extension to the work presented in this paper.

\section{ACKNOWLEDGMENTS}

The authors thank Andhra University, Visakhapatnam, India, where they have put up more than twenty five years of service in teaching, for having provided conducive environment for academics and research thus giving scope for continuous learning.

\section{APPENDIX}

\section{To Take Linear Term As Approximation For}

$\ln \mathbf{Z}$ (Sections 3.1 and 3.2 of the paper have reference to this)

Since for any real number $z$ that satisfies $0<z<2$, the following formula holds:

$\ln z=(z-1)-\frac{(z-1)^{2}}{2}+\frac{(z-1)^{3}}{3}-\frac{(z-1)^{4}}{4}+\cdots$

Taking linear term as approximation,

$\ln z=(z-1)$

$$
\text { (A1) }
$$

\section{To Prove That $\frac{\partial \log B\left(1 / 2, v_{l} / 2\right)}{\partial v_{l}}=\frac{-1}{2\left(v_{l}+1\right)}$}

(Section 3.3 of the paper has reference to this)

We know that $\frac{\partial \log B\left(1 / 2, v_{l} / 2\right)}{\partial v_{l}}=\frac{\frac{\partial}{\partial v_{l}} B\left(1 / 2, v_{l} / 2\right)}{B\left(1 / 2, v_{l} / 2\right)}$

Since we know that beta function $B(a, b)$ is defined as 
$B(a, b)=\int_{0}^{1} x^{a-1}(1-x)^{b-1} d x \quad$ for $\quad a>0, b>0$

(A2)

$\frac{\partial}{\partial v_{l}} B\left(1 / 2, v_{l} / 2\right)=\int_{0}^{1} \frac{\partial}{\partial v_{l}}(1-x)^{-1 / 2} x^{\left(v_{l}-2\right) / 2} d x$

To solve the above equation, we have to first solve $\frac{\partial}{\partial v_{l}} x^{\left(v_{l}-2\right) / 2}$

Let $\quad y=x^{\left(v_{l}-2\right) / 2} \Rightarrow \log y=\log x^{\left(v_{l}-2\right) / 2}=$

$\frac{\left(v_{l}-2\right)}{2} \log x$

$\Rightarrow \frac{1}{y} d y=\frac{\log x}{2} d v_{l} \Rightarrow \frac{d y}{d v_{l}}=y \frac{\log x}{2}$

$\therefore \frac{\partial}{\partial v_{l}} x^{\left(v_{l}-2\right) / 2}=x^{\left(v_{l}-2\right) / 2}\left(\frac{\log x}{2}\right)$

Hence Equation (A3) can be written as

$\frac{\partial}{\partial v_{l}} B\left(1 / 2, v_{l} / 2\right)=\int_{0}^{1} x^{\left(v_{l}-2\right) / 2}\left(\frac{\log x}{2}\right)(1-x)^{-1 / 2} d x$

Taking linear term as approximation of $\log x$ as $(x-1)$, given by Equation (A1), the above equation becomes

$$
\begin{gathered}
\frac{\partial}{\partial v_{l}} B\left(1 / 2, v_{l} / 2\right)=\int_{0}^{1} x^{\left(v_{l}-2\right) / 2}\left(\frac{x-1}{2}\right)(1-x)^{-1 / 2} d x \\
\Rightarrow-\frac{1}{2} \int_{0}^{1} x^{\left(v_{l}-2\right) / 2}(1-x)^{-1 / 2} d x \\
\Rightarrow-\frac{1}{2} \int_{0}^{1} x^{\frac{v_{l}}{2}-1}(1-x)^{\frac{3}{2}-1} d x
\end{gathered}
$$

$\Rightarrow-\frac{1}{2} B\left(v_{l} / 2,3 / 2\right)$

(Section 3.3

of

the paper has reference to this)

$\therefore \frac{\partial}{\partial v_{l}} B\left(1 / 2, v_{l} / 2\right)=-\frac{1}{2} B\left(v_{l} / 2,3 / 2\right)$

(A4)

$$
\text { Hence } \quad \frac{\partial \log B\left(1 / 2, v_{l} / 2\right)}{\partial v_{l}}=\frac{\frac{\partial}{\partial v_{l}} B\left(1 / 2, v_{l} / 2\right)}{B\left(1 / 2, v_{l} / 2\right)}=
$$

$\frac{-\frac{1}{2} B\left(v_{l} / 2,3 / 2\right)}{B\left(1 / 2, v_{l} / 2\right)}$

$=-\frac{1}{2} \frac{\Gamma\left(\frac{v_{l}}{2}\right) \Gamma\left(\frac{3}{2}\right)}{\Gamma\left(\frac{v_{l}}{2}+\frac{3}{2}\right)} \frac{\Gamma\left(\frac{v_{l}}{2}+\frac{3}{2}\right)}{\Gamma\left(\frac{v_{l}}{2}\right) \Gamma\left(\frac{1}{2}\right)}=-\frac{1}{2} \frac{\Gamma\left(\frac{1}{2}+1\right)}{\Gamma\left(\frac{1}{2}\right)} \frac{\Gamma\left(\frac{v_{l}}{2}+\frac{1}{2}\right)}{\Gamma\left(\frac{v_{l}}{2}+\frac{1}{2}+1\right)}=$

$-\frac{1}{2} \frac{\frac{1}{2} \Gamma\left(\frac{1}{2}\right)}{\Gamma\left(\frac{1}{2}\right)} \frac{\Gamma\left(\frac{v_{l}}{2}+\frac{1}{2}\right)}{\frac{\left(v_{l}+1\right)}{2} \Gamma\left(\frac{v_{l}}{2}+\frac{1}{2}\right)}=\frac{-1}{2\left(v_{l}+1\right)}$

Since $B(a, b)=B(b, a)=\frac{\Gamma(a) \Gamma(b)}{\Gamma(a+b)}$

and the gamma function, denoted by $Г($.$) , is defined by$

$\Gamma(t)=\int_{0}^{\infty} x^{(t-1)} e^{-x} d x \quad$ for $t>0$

(A6)

The following definitions about gamma function are taken from [5] as given by Mood et al.

$\Gamma(t)$ is nothing more than a notation for the definite integral that appears on the right hand side of Equation (6). Integration by parts yields

$\Gamma(t+1)=t \Gamma(t)$

(A7) and hence, if $t=n($ an integer $), \Gamma(n+1)=n !$

If $n$ is an integer,

$\Gamma\left(n+\frac{1}{2}\right)=\frac{1.3 \cdot 5 \ldots(2 n-1)}{2^{n}} \sqrt{\pi}$, and in particular $\Gamma\left(\frac{1}{2}\right)=$ $2 \Gamma\left(\frac{3}{2}\right)=\sqrt{\pi}$.

III. To Derive Partial Derivatives Of Incomplete

Beta Functions With Respect To $\mu_{l}, c_{l}$, and $v_{l}$.

Here, the partial derivatives of incomplete beta functions with respect to $\mu_{l}, c_{l}$, and $v_{l}$ for solving Equations (18), (19), (20) related to the truncated normal with gamma mixture distribution have been derived for the purpose of their use in sections $3.1,3.2$, and 3.3 of the paper.

$b_{1}=\frac{c}{c+(b-\mu)^{2}}$ and $a_{1}=\frac{c}{c+(a-\mu)^{2}}$ since $t=\frac{c}{c+(x-\mu)^{2}}$, details of which can be seen sections 5.2 and 5.3 in the said chapter.

$\frac{\partial}{\partial \mu_{l}} B_{a_{1}}\left(\frac{v_{l}}{2}, \frac{1}{2}\right)=\frac{\partial}{\partial \mu_{l}} \int_{0}^{a_{1}} y^{\frac{v_{l}}{2}-1}(1-y)^{\frac{1}{2}-1} d y$

$=\frac{\partial}{\partial \mu_{l}} \int_{0}^{\frac{c_{l}}{c_{l}+\left(a-\mu_{l}\right)^{2}}} y^{\frac{v_{l}}{2}-1}(1-y)^{\frac{1}{2}-1} d y$

(A8)

The above equation is in the form of $\frac{\partial}{\partial x} \int_{0}^{\psi(x)} f(x) d x$, which is equal to $f(\psi(x)) \psi^{\prime}(x)$

Therefore, the solution for Equation (A8) is

$\left[\frac{c_{l}}{c_{l}+\left(a-\mu_{l}\right)^{2}}\right]^{\frac{v_{l}}{2}-1}\left[1-\frac{c_{l}}{c_{l}+\left(a-\mu_{l}\right)^{2}}\right]^{-\frac{1}{2}} \frac{d}{d \mu_{l}}\left[\frac{c_{l}}{c_{l}+\left(a-\mu_{l}\right)^{2}}\right]$

$\Rightarrow\left[\frac{c_{l}}{c_{l}+\left(a-\mu_{l}\right)^{2}}\right]^{\frac{v_{l}}{2}-1}\left[1-\frac{c_{l}}{c_{l}+\left(a-\mu_{l}\right)^{2}}\right]^{-\frac{1}{2}} \frac{2 c_{l}\left(a-\mu_{l}\right)}{\left[c_{l}+\left(a-\mu_{l}\right)^{2}\right]^{2}}$

since

$\frac{d}{d \mu_{l}}\left[\frac{c_{l}}{c_{l}+\left(a-\mu_{l}\right)^{2}}\right]=\frac{c_{l}^{\prime}\left[c_{l}+\left(a-\mu_{l}\right)^{2}\right]-c_{l}\left[c_{l}+\left(a-\mu_{l}\right)^{2}\right]^{\prime}}{\left[c_{l}+\left(a-\mu_{l}\right)^{2}\right]^{2}}=\frac{2 c_{l}\left(a-\mu_{l}\right)}{\left[c_{l}+\left(a-\mu_{l}\right)^{2}\right]^{2}}$

Upon simplification, the above equation may be written as

$\frac{\partial}{\partial \mu_{l}} B_{a_{1}}\left(\frac{v_{l}}{2}, \frac{1}{2}\right)=\frac{2}{c_{l}{ }^{1 / 2}}\left[\frac{c_{l}}{c_{l}+\left(a-\mu_{l}\right)^{2}}\right]^{\frac{v_{l}+1}{2}}$

Similarly

$\frac{\partial}{\partial c_{l}} B_{a_{1}}\left(\frac{v_{l}}{2}, \frac{1}{2}\right)=$

$\left[\frac{c_{l}}{c_{l}+\left(a-\mu_{l}\right)^{2}}\right]^{\frac{v_{l}}{2}-1}\left[1-\frac{c_{l}}{c_{l}+\left(a-\mu_{l}\right)^{2}}\right]^{-\frac{1}{2}} \frac{d}{d c_{l}}\left[\frac{c_{l}}{c_{l}+\left(a-\mu_{l}\right)^{2}}\right]$

$\Rightarrow\left[\frac{c_{l}}{c_{l}+\left(a-\mu_{l}\right)^{2}}\right]^{\frac{v_{l}}{2}-1}\left[1-\frac{c_{l}}{c_{l}+\left(a-\mu_{l}\right)^{2}}\right]^{-\frac{1}{2}} \frac{\left(a-\mu_{l}\right)^{2}}{\left[c_{l}+\left(a-\mu_{l}\right)^{2}\right]^{2}}$

since

$\frac{d}{d c_{l}}\left[\frac{c_{l}}{c_{l}+\left(a-\mu_{l}\right)^{2}}\right]=\frac{c_{l}^{\prime}\left[c_{l}+\left(a-\mu_{l}\right)^{2}\right]-c_{l}\left[c_{l}+\left(a-\mu_{l}\right)^{2}\right]^{\prime}}{\left[c_{l}+\left(a-\mu_{l}\right)^{2}\right]^{2}}=\frac{\left(a-\mu_{l}\right)^{2}}{\left[c_{l}+\left(a-\mu_{l}\right)^{2}\right]^{2}}$

Upon simplification, the above equation may be written as

$\frac{\partial}{\partial c_{l}} B_{a_{1}}\left(\frac{v_{l}}{2}, \frac{1}{2}\right)=\frac{\left(a-\mu_{l}\right)}{c_{l} c_{l} 1 / 2}\left[\frac{c_{l}}{c_{l}+\left(a-\mu_{l}\right)^{2}}\right]^{\frac{v_{l}+1}{2}}$

(A10)

In respect of $\frac{\partial}{\partial v_{l}} B_{a_{1}}\left(\frac{v_{l}}{2}, \frac{1}{2}\right)$, Equation (A4) holds good.

$\therefore \frac{\partial}{\partial v_{l}} B_{a_{1}}\left(\frac{v_{l}}{2}, \frac{1}{2}\right)=-\frac{1}{2} B_{a_{1}}\left(v_{l} / 2,3 / 2\right)$

(A11) 


\section{REFERENCES}

[1] Viziananda Row Sanapala, Sreenivasa Rao Kraleti, and Srinivasa Rao Peri. Image Segmentation Using Compound Normal with Gamma Mixture Model, International Journal of Computer Science Issues, Volume 12, Issue 4, July 2015, ISSN (Print): 1694-0814 | ISSN (Online): 1694-0784, www.IJCSI.org.

[2] S. Viziananda Row, Image Segmentation Using Compound Normal with Gamma Mixture Model and its Truncated Version, Ph. D. Thesis, Andhra University, Visakhapatnam, India, 2016.

[3] Normal L. Johnson, Samuel Kotz and N. Balakrishnan. Continuous Univariate Distributions, Vol-I , Second Edition. John Wiley\&Sons, 2007.

[4] https://en.wikipedia.org/wiki/Truncated_distribution

[5] Alexander M. Mood, Franklin A. Graybill, and Duane C. Boes. Introduction to the theory of Statistics, Tata McGraw-Hill, Third Edition, 2001.

[6] T. Lei and J. K. Udupa. Performance evaluation of finite normal mixture model-based image segmentaion techniques. IEEE Transactions on Image Processing, vol 12, no 10, pp. 1153-1169, 2003.
[7] J. Zhang and J. M. Modestino. A model fitting approach to cluster validity with application to stochastic model based image segmentation, IEEE Transactions on Pattern Analysis and Machine Intelligence, vol. 12, no. 10, pp.1009-1016, 1990.

[8] T. Lei and W. Sewchand. Statistical approach to X-ray CT imaging and its applications in image analysis-Part 2: A new stochastic model based image segmentation technique for CT image, IEEE Transactions on Medical Imaging, vol. 11, no. 1, pp. 62-69, 1992.

[9] Z. Liang and J. R. MacFall. "Parameter estimation and tissue segmentation of multispectral MR images." IEEE Transactions on Medical Imaging, vol. 13, no.3, pp. 441-449, 1994.

[10] A. P. Dempster, N. M. Laird and D. B. Rubin Maximum-likelihood from incomplete data via the EM algorithm, J. Royal Statist. Soc. Ser. B (methodological), 39 (1977), pp. 1-38.

[11] J. A. Bilmes. "A gentle tutorial of the EM algorithm and its application to parameter estimation for Gaussian mixture and hidden Markov models." International Computer Science Institute, Berkely CA, 94704, pp 1-13. 